\title{
Field-controlled Self-assembly and Disassembly of Colloidal Nanoparticles
}

\author{
Marco Lattuada*, Marco Furlan, and Yogesh Harshe
}

\begin{abstract}
Self-assembly of nanoparticles is one of the most promising methods for the preparation of novel materials and devices with exceptional properties. In order to control nanoparticles self-assembly, an understanding of their interactions is absolutely necessary. One convenient way to achieve a control on their interaction is through the use of external fields. Here we provide two different examples of how interparticle interactions are affected by interactions with external fields. In the first case, magnetic fields are used to induce dipolar interactions among concentrated suspension of superparamagnetic nanocolloids, which cause them to self-assemble into dense chain-like anisotropic structures, used as templates for the growth of porous materials with tunable properties. In the second case, it is shown how more commonly employed but less understood flow fields interact with clusters of particles, and lead to their restructuring or disassembly depending upon the shear stress applied.
\end{abstract}

Keywords: Cluster disassembly · Flow fields · Magnetic fields · Nanoparticle self-assembly ·

Porous materials

\section{Introduction}

One of the primary objectives of researchers in the field of nanotechnology is the creation of materials and structures with precise control of their architecture at the nanoscale. ${ }^{[1]}$ Among the different strategies to achieve this goal, self-assembly of well-defined nano building-blocks is one of the most sought after. Self-assembly means that, in principle, aptly engineered 'nano-bricks' with accurately designed properties (such as size, shape, interactions, composition, etc.) could spontaneously organize themselves into objects and ideally into devices with a length scale ranging from nanometers to macroscopic sizes, and with desired structures and functionalities..$^{[2]}$ Nevertheless, considerable hurdles still need to be overcome before scientists and engineers will be finally

${ }^{*}$ Correspondence: Dr. M. Lattuada ETH Zürich

Department of Chemistry and Applied Biosciences Institute for Chemical and Bioengineering

Wolfgang Pauli Strasse 10

$\mathrm{CH}-8093$ Zürich

Tel.: +4144632 0712

Fax: +41 446321082

E-mail: marco.lattuada@chem.ethz.ch able to fully exploit the potentials of selfassembly. The challenges ahead of scientists are both experimental and theoretical. From the experimental point of view, novel and efficient techniques are required to produce sufficiently large amounts of nano building-blocks featuring controlled size, shape, structure, and functionalities at a reasonable cost. From the theoretical view point, there is the need to better understand the relationship between interactions at the nanoscale, self-assembly kinetics and thermodynamics of nano building-blocks..$^{[1]}$

In order to fully exploit the potential of self-assembly, it is of key importance that the interactions among nanoparticles are well understood, since interparticle interactions are the driving force for self-assembly. While some interparticle interactions, such as Van der Waals, electrostatic and steric interactions, are well known and characterized, they usually do not allow one to obtain a wide variety of structures, but only isotropic disordered assemblies or colloidal crystals. ${ }^{[3]}$ In order to expand the possibilities offered by self-assembly, two approaches are conceivable. On one hand, nanoparticles with more complex architecture, such as Janus or patched morphology need to be created. ${ }^{[4]}$ Creating nanoparticles capable of forming bonds only in specific orientations, similar to molecules, would certainly increase the range of accessible structures that can be obtained through self-assembly. On the other hand, the application of external fields that can modulate the interactions among nanoparticles can also have a profound effect on their assembly characteristics. Several examples of this last strategy are worth mentioning. Electric fields are commonly used to move charged nanoparticles and assemble them into ordered structures, and also to induce dipolar interaction in dielectric particles. ${ }^{[5]}$ Magnetic fields can be utilized both to move magnetic nanoparticles, to align into chain-like structures and to separate them from non-magnetic particles. ${ }^{[6]}$ Gravitational and centrifugal fields are commonly used to fractionate suspensions of particles with different sizes and/or densities. ${ }^{[1]}$ Flow fields are used to transport and fractionate particles, to enhance aggregation as well as to disperse particles and disassemble clusters thereof. ${ }^{[7]}$ A major advantage of controlling nanoparticle assembly and disassembly with certain external fields is that these can be easily turned on and off on demand, thus resulting in a powerful tool to control and affect on demand nanoparticle interactions.

In this work, we would like to show some of the possibilities offered by studying nanoparticle interactions with external fields. We have selected two very different examples, one predominantly experimental, the other computational. The first one aims at demonstrating how superparamagnetic nanocolloids can interact with magnetic fields, developing on demand dipolar interactions, with the objective to magnetically control the structure of porous materials. On the other hand, the second example targets the interactions of particles with flow fields, which are less 'exotic' and more commonly used in industrial processes, but far less understood, with the goal to show how computations can help us understand the interactions between nanoparticles under the influence of flow fields, and in particular how such fields can disassemble clusters of nanoparticles. 


\section{Experimental Part}

\section{Materials}

Divinylbenzene technical grade (DVB), 2,2'-azobis-isobutyronitrile purum $\geq 98 \%$ (AIBN) and diethyl ether puriss, n-dodecanol $>99.5 \%$, urease from jack beans $30.0 \mathrm{U} / \mathrm{mg}$ and poly(ethylene) glycol (PEG) 10kDa, analytical standard, were obtained from Fluka. Urea pro analisis and ammonium solution for analysis $(25 \mathrm{wt} \%)$ were obtained from Merck. Iron(II) chloride tetrahydrate Reagent-Plus $99 \%$, ricinoleic acid technical grade $>80 \%$ (RA) and acetone spectrophotometric $\geq 99 \%$ were obtained from Sigma-Aldrich. Iron(III) chloride hexahydrate extra pure $99+\%$ and $n$-hexadecane $99 \%$ (HD) were obtained from Acros Organics. Styrene general purpose grade $(\mathrm{St})$ was obtained from Fisher Scientific. Tetramethoxysilane 98\% (TMOS) was obtained from ABCR. Acetic acid glacial was obtained from Carlo Erba reagents. Potassium persulfate (KPS) $99.0 \%$ min was obtained from Alfa Aesar. Pluronic F68 was obtained from Applichem. Ethanol absolute, analytical grade, was obtained from Scharlau. Sodium monolaurylmaleate surfactant was synthesized as reported in the literature. ${ }^{[6 a]}$ If not specified otherwise, all chemicals were used as obtained.

\section{Synthesis of Magnetite Nanocrystals}

Oil-soluble magnetite nanocrystals were produced via a modification of the coprecipitation method developed by Massart. ${ }^{[8]}$ In a typical reaction $3.90 \mathrm{~g}$ $\mathrm{FeCl}_{2} \cdot 4 \mathrm{H}_{2} \mathrm{O}$ and $10.71 \mathrm{~g} \mathrm{FeCl}_{3} \cdot 6 \mathrm{H}_{2} \mathrm{O}$ were mixed in $180 \mathrm{ml} \mathrm{H}$ O. Once the salts were completely dissolved a mixture composed of $8.56 \mathrm{~g}$ ricinoleic acid and $4.8 \mathrm{~g}$ acetone was added. Then, the solution was heated up to $80^{\circ} \mathrm{C}$, followed by the addition of 27 $\mathrm{ml} \mathrm{NH}$. The reaction was carried out for $30 \mathrm{~min}$, then the solution was cooled down to room temperature. The product was then precipitated in acetone, washed three times with water and acetone and then dried for $12 \mathrm{~h}$. The product was subsequently redispersed in diethyl ether, filtrated magnetically and dried in rota-vapor by $40{ }^{\circ} \mathrm{C}$ and 700 mbar.

\section{Synthesis of Magnetic Nanocolloids}

In order to obtain sterically-stabilized magnetic nanocolloids, $3 \mathrm{~g}$ of the abovesynthesized magnetite nanocrystals were dispersed in $5.4 \mathrm{~g}$ styrene, $0.6 \mathrm{~g}$ divinyl benzene, $0.125 \mathrm{~g}$ hexadecane and $0.06 \mathrm{~g}$ AIBN. The obtained oil phase was then mixed with a solution composed of $48 \mathrm{~g}$ water and $0.60 \mathrm{~g}$ Pluronic F68. In the case of electrostatically-stabilized magnetic colloids, an analogous procedure was used, except that the oil phase was obtained by mixing $7.98 \mathrm{~g}$ styrene, $2.9 \mathrm{~g}$ diethyl ether, $0.42 \mathrm{~g}$ divinylbenzene, $0.25 \mathrm{~g}$ hexadecane and $8.4 \mathrm{~g}$ magnetite nanocrystals, while the aqueous phase was composed of $48 \mathrm{~g}$ water and $0.5 \mathrm{~g}$ sodium monolaurylmaleate surfactant. The obtained solution was sonicated using a Branson Digital Sonifier S-450D for $15 \mathrm{~min}$ at $35 \%$ amplitude with a duty cycle of 0.5 seconds. The miniemulsion was then transferred to a three-neck flask, flushed with $\mathrm{N}_{2}$ for 5 min and then heated up to $70{ }^{\circ} \mathrm{C}$ for $5 \mathrm{~h}$, at which point almost complete conversion of the monomer was reached.

\section{Magnetic Gelation of Electro- statically Stabilized Nanoparticles}

The gelation process follows the method developed by Gauckler et al. ${ }^{[9]}$ A stock swelling solution was prepared by mixing $9 \mathrm{~g}$ styrene, $1 \mathrm{~g}$ divinylbenzene and $0.1 \mathrm{~g}$ AIBN. The magnetic nanocolloids suspension was first swollen with an amount of swelling solution comprised between $20-40 \mathrm{wt} \%$ of the dry fraction of the latex, which was added dropwise and mixed for $4 \mathrm{~h}$. Afterwards, $1 \mathrm{ml}$ of the swollen latex was mixed with $0.5 \mathrm{ml}$ urea solution (4 M) and $0.5 \mathrm{ml}$ urease solution (960 units $/ \mathrm{ml}$ ). When the gelation was carried out in the presence of a magnetic field, the sample holder was positioned in between the poles of a large permanent magnet, which generates a magnetic field with an intensity of 1 $\mathrm{T}$. The final solid weight percentage in the gel was $9.5 \%$, corresponding to a particle volume fraction of $7 \%$. After gelation was completed, the temperature was increased to $70{ }^{\circ} \mathrm{C}$ by means of a heating jacket and a post-polymerization was carried out for $24 \mathrm{~h}$, when required in the presence of the magnetic field.

\section{Synthesis of Silica Monoliths}

The synthesis of the silica monoliths is based on a modification of a recipe described elsewhere. ${ }^{[10]}$ In a typical experiment, 0.29 g PEG (10kDa), $2.85 \mathrm{ml}$ acetic acid $0.01 \mathrm{M}$ and $1 \mathrm{ml}$ TMOS were mixed together at $1250 \mathrm{rpm}$ at $0{ }^{\circ} \mathrm{C}$ for $30 \mathrm{~min}$, then $2.85 \mathrm{ml}$ of the above prepared sterically stabilized latex and $0.0228 \mathrm{ml}$ of acetic acid 1.25 M were added. The mixture was then stirred for $2 \mathrm{~min}$ and then poured in two molds, one of which was put in an oven at $40{ }^{\circ} \mathrm{C}$, while the second one was put in a heat jacket inside a magnetic field of $1 \mathrm{~T}$ at the temperature of $40{ }^{\circ} \mathrm{C}$. Both samples were cured for $12 \mathrm{~h}$. The obtained monoliths were first immersed in a solution of water-ethanol 1:1 by volume for 24 $\mathrm{h}$ and then in pure ethanol for additional $48 \mathrm{~h}$. In order to remove all the water, the ethanol solution was replaced with fresh one every $12 \mathrm{~h}$. The monoliths were then dried in an oven at $50{ }^{\circ} \mathrm{C}$ for 3 days and finally calcined at $600{ }^{\circ} \mathrm{C}$ for $3 \mathrm{~h}$.

\section{Computational Part}

\section{Stokesian Dynamic Simulations}

The Stokesian dynamics (SD) method was developed by Brady and Bossis as a powerful computational technique to account for hydrodynamic interactions in concentrated suspensions of spherical particles in the presence of shear. ${ }^{[11]}$ The method has been successfully used to describe the complex rheological properties of particles suspensions. The basic assumptions underlying SD are: i) particle Reynolds numbers should be small, so that Stokes equations can be used to describe particles-fluid interactions $\left(\operatorname{Re}_{\mathrm{p}}<<1\right)$; ii) the shear rate has to be constant over the simulation length scale; iii) fluid relaxation times should be much shorter than particles relation times. The basic equation of SD is an extended form of Stokes law, i.e. a linear relationship between relative translational velocities, relative angular velocities, $\Omega_{n}-\Omega^{\infty}$, and shear rate tensor $E^{\infty}$ and forces $F_{n}{ }_{n}$, torques, $T_{n}$ and stresslets $S_{n}$, acting on all particles in the system, which in matrix form can be written as follows:

$$
\left[\begin{array}{l}
F_{n} \\
T_{n} \\
S_{n}
\end{array}\right]=R \cdot\left[\begin{array}{l}
U_{n}-U^{\infty} \\
\Omega_{n}-\Omega^{\infty} \\
-E^{\infty}
\end{array}\right]
$$

Here $R$ stands for the grand resistance matrix, having a size equal to $11 N_{\text {sphere }} \times$ $11 N_{\text {sphere }}$ with $N_{\text {sphere }}$ being the number of particles in the simulation domain. The knowledge of the grand resistance matrix, which is only a function of the relative position of the particles, and accounts for their hydrodynamic interactions, permits the computation of all particles velocities and thus of their trajectories if all the external and interparticle forces acting on them are known. Unfortunately, an exact calculation of $R$ is not possible except for two particles.[11b] Therefore, Brady and Bossis approximated the grand resistance matrix, by combining two approaches, one capable of accurately accounting for the far-field many-body interactions, the other one accounting for the lubrication forces that dominate the interactions among particles when they are nearly touching. ${ }^{[11 b]}$ The reader is advised to consult relevant literature for further details, which are outside the scope of this article. ${ }^{[11 b]}$ A typical SD simulation aims to track all particle positions as a function of time, by inverting Eqn. (1), and then solving Newton's equation of motion, assuming that particle inertia is negligible. The solution of the equations of motion is a computationally expensive procedure, requiring frequent calculations and inversion of the large grand resistance matrix.

Apart from the hydrodynamic interac- 
tions, which are transmitted through the fluid in which the particles are suspended, the particles experience different attractive and repulsive forces. In the case of colloidal particles, particle-particle interactions are usually dominated by short range attractive Van der Waals interactions and by Born repulsion, introduced to prevent overlap between closely separated particles. ${ }^{[12]}$ Recently, Pantina and Furst ${ }^{[13]}$ experimentally demonstrated that the dynamics of chains of particles cannot be accurately modeled without accounting for the presence of tangential interactions among particles in contact, which are responsible for imparting bending rigidity to interparticle bonds. To take this phenomenon into account, we have followed the methodology developed by Becker and Briesen, ${ }^{[14]}$ which models the contact forces between particles separated by very short distances primarily by using elastic and friction contributions. The numerical implementation of tangential forces was performed using the Discrete Element Method (DEM) of Cundall and Strack. ${ }^{[15]}$ To integrate the motion equations, Runge-Kutta $4^{\text {th }}$ order method was used. At each time step the particle-particle interactions were updated and contacts were identified.

All the simulations performed started with a number $N_{\text {spheres }}$ of identical particles with radius $R_{p}$ assembled into a single cluster. The size of cluster, i.e. its radius of gyration $R$, is always related to its mass by means of a typical fractal scaling equation:[16]

$$
N_{\text {spheres }}=k\left(\frac{R_{g}}{R_{p}}\right)^{d_{f}}
$$

where $d_{f}$ is the cluster fractal dimension $\left(1.8 \leq d_{f} \leq 3\right.$ in this work) and $k$ is a constant with a value close to 1 .

\section{Results and Discussion}

\section{Magnetically-driven Self-assembly}

The first part of this work is primarily experimental and devoted to show how external magnetic fields can be used to manipulate the interactions among magnetic nanoparticles and drastically widen the range of structures that can be created from of their self-assembly. It is common knowledge that magnetic particles can develop strong dipolar interactions. [6c] When ferromagnetic materials are utilized, particles behave as small permanent magnets, and form clusters with stringlike morphology, affecting the colloidal stability of the suspension. On the other hand, small magnetic nanoparticles with a size below a critical threshold, which in the case of magnetite is $\sim 20 \mathrm{~nm}$, become superparamagnetic. This means that dipolar interactions manifest themselves only in the presence of magnetic field, while in the absence of a field a suspension of superparamagnetic nanocrystals behaves like a typical non-magnetic suspension of nanoparticles.[6c,17]

Due to the low strength of dipolar interactions of small superparamagnetic nanocrystals, we decided to work with polymer-magnetite nanocolloids prepared by miniemulsion polymerization, having a size range of about $90-150 \mathrm{~nm}$, in which a large number of hydrophobically modified 5-15 nm superparamagnetic magnetite nanocrystals were incorporated.[6a] Vibration-sample magnetometer measurements of the magnetization behavior of these magnetic nanocolloids show that they maintain superparamagnetism, despite being able to develop strong dipolar interactions in the presence of an applied magnetic field.

We will first show how magnetic fields can be utilized to drastically change the structure of colloidal gels made of our magnetic colloids. Colloidal gels are porous disordered structures obtained from self-assembly of nanoparticles in the presence of strong isotropic attractive interactions. Gels are out-of-equilibrium structures, formed from the percolation of clusters of nanoparticles with a fractal structure, commonly obtained in the presence of either Van der Waals or depletion interactions. ${ }^{[18]}$ In the case of electrostatically stabilized nanocolloids, like the ones we have prepared, gelation can be induced by screening the electrostatic repulsive interactions arising from surface charges through a sufficient increase of the ionic strength of the suspension. Since in colloidal gels the nanoparticles are held together by non-covalent interactions, their mechanical properties are often very poor. For this reason we have exploited a postpolymerization procedure to harden the gels. ${ }^{[6 a]}$ Fig. 1a shows an SEM picture of a colloidal gel obtained by self-assembly of charged-stabilized magnetic nanocolloids in the absence of an external magnetic field, with a particle volume fraction of about $7 \%$. One can appreciate how the structure of such a gel is disordered and isotropic, featuring dense interconnected clusters of nanoparticles and large holes.

In order to manipulate the final structure of the gels, we have performed colloidal gelation of magnetic nanocolloids in the presence of a magnetic field. An example of the typical structure that can be obtained in this manner is shown in Fig. 1b. One can observe how the structure is no longer a disordered assembly of particles, but is instead made of bundles of nanoparticles primarily aligned in one direction, i.e. that of the applied field, while
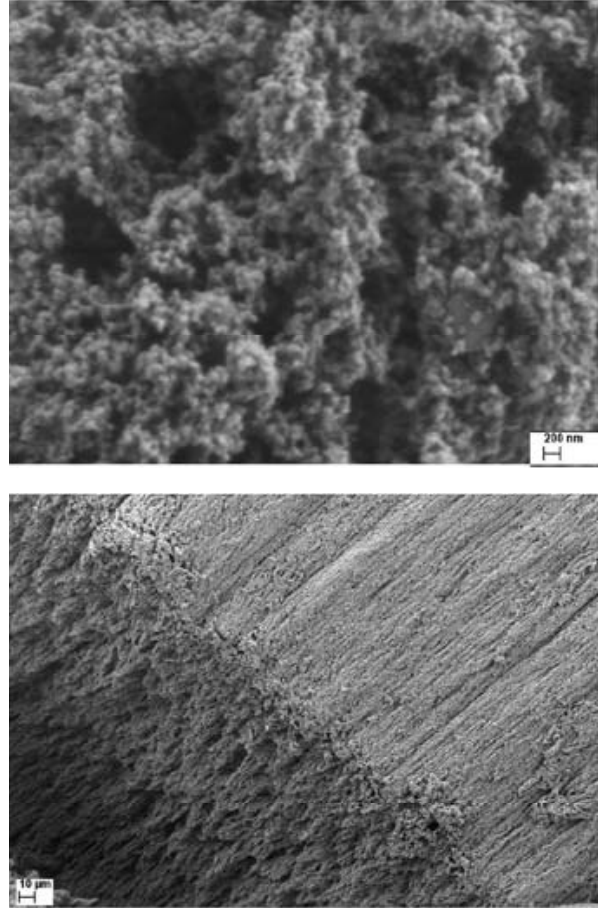

Fig. 1. SEM picture of a colloidal gel obtained from self-assembly of magnetic nanocolloids in the absence (top) and the presence (bottom) of an external magnetic field.

still maintaining a disordered structure in any perpendicular direction. A closer look at the structure also reveals that, within the chain-like structures, the nanoparticles are not perfectly aligned, but maintain a certain extent of disorder. Since nanocolloids are not perfectly monodisperse, both in size and in the amount of encapsulated magnetite per particle, there is a distribution of magnetic moments and in dipolar interactions within the suspension. Additionally, there is a competition between magnetic interactions and diffusion in driving the formation of the gel. While the former driving force tends to align particles, the latter has a shuffling effect, which leads to more disordered structures. Clearly, the stronger the dipolar interactions, the more pronounced is the alignment of the nanocolloids in the gel. Our investigations have in fact demonstrated that the degree of anisotropy of the gels, as measured by means of magnetic torque, can be controlled by tuning the strengths of dipolar interactions among the colloids, by reducing the intensity of the applied field or by decreasing the magnetization of each nanocolloid.[6a]

We have also started using the peculiar structures formed by magnetic nanocolloids in the presence of a magnetic field as templates for the preparation of ceramicbased materials via the sol-gel method. In a conventional sol-gel method, a silicon precursor is dispersed in an aqueous solution containing a low amount of acid, which acts as a catalyst for the nucleation of silica nanoparticles. The particles continue to 
grow and eventually interconnect into a gel network. In order to control the porosity of final gel, a porogen, i.e. a polymer incompatible with silica, is often added to the solution (usually PEG). ${ }^{[10]}$ The porogen accumulates in certain regions in the gel, preventing a homogeneous growth of silica, leading to the formation of a network of silica microspheres with large interconnected pores. We have partially substituted the porogen with our magnetic nanocolloids. The nanocolloids are therefore dispersed in a mixture of acetic acid solution, PEG and pre-hydrolyzed silicon precursor (TMOS). In this solution their stability is guaranteed by the presence of the steric surfactant pluronics F68. The silica gel grows around the particles, which act as shape-directing agents. In the absence of a magnetic field, a structure similar to that of a conventional colloid gel is formed, with silica covering clusters of nanocolloids and linking them together until a monolith is formed.

In the presence of a magnetic field, the polymer particles are grouped into bundles, aligned in the same direction of the magnetic field, and then covered by silica, as Fig. 2 shows. Since the alignment process is practically instantaneous, whereas the sol-gel process takes a longer time, depending on the acid concentration, the silica starts to deposit on the particles when they are already aligned. At the end of the process the monolith is hardened by calcination at $600{ }^{\circ} \mathrm{C}$, where the polymer is burned away, leading to an intact silica monolith with some magnetite nanocrystals dispersed in it.

The microstructural changes in the porous materials lead to significant alterations of the material properties. First of all, the porosity of the material can be varied with the application of a magnetic field. In Fig. $3 \mathrm{a}$ we see how the porosity of a monolith, measured by mercury intrusion porosimetry, changes depending on whether the monolith has been prepared in the presence or in the absence of a magnetic field. In the

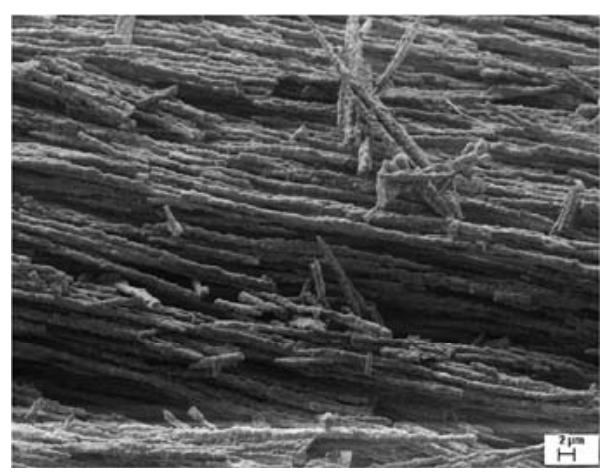

Fig. 2. SEM picture of a silica gel obtained from a sol-gel process with a porogen (PEG) and magnetic nanocolloids, prepared in the presence of an external magnetic field. absence of a field, the pore size distribution shows two peaks, one at around 100 $\mathrm{nm}$, and a second much broader one at 1 $\mu \mathrm{m}$. In the presence of a field, instead, the peak corresponding to the smallest pore size is shifted to lower sizes, while the second peak has disappeared and has been replaced by a broad shoulder of the first peak. A drastic change in the mechanical properties of the materials is also observed. Fig. $3 b$ shows the result of mechanical compressive tests run on an isotropic sample prepared in the absence of a field, and an identical anisotropic one prepared in the presence of a field. The latter has been compressed along two directions, one parallel and one perpendicular to the applied magnetic field. It can be observed how this sample exhibits a strong mechanical anisotropy, with a maximum compressive stress one order of magnitude higher in the direction of the field than in the perpendicular one, and five times higher than the isotropic sample. These results show how effectively both structural and mechanical properties of porous materials can be controlled through the application of an external magnetic field in the presence of magnetic nanocolloids.

\section{Flow-field Induced Disassembly of Colloidal Clusters}

The second part of our discussion focuses on how simulations can help us to unveil how colloidal particles interact under flow fields. Although the use of flow fields is common in processes of industrial relevance, for example for controlled coagulation of polymer latexes or in pharmaceutical processes, the interactions of particles and clusters thereof with flow fields is a very poorly understood phenomenon. The complexity in deciphering such a process arises both because typical clusters of nanoparticles have a complex structure, and also due to the multi-body nature of hydrodynamic interactions. SD simulations can help us to quantify this process. ${ }^{[12]}$ In order to start from realistic but well-defined conditions, we have created a large database of clusters spanning a broad range of clusters masses and fractal dimension values, covering both those clusters created by assembly of particles driven by diffusion, which have an open structure (low fractal dimension) and clusters obtained by the assembly of particles in the presence of shear forces, which instead have a denser packing (high fractal dimension), ${ }^{[16,19]}$ respectively. The aspect that is of particular interest is the fate of an isolated cluster exposed to well-defined simple shear conditions. There are three scenarios that can be investigated. When shear forces are sufficiently stronger than interparticle forces keeping the particles together, the applied flow field will disassemble the cluster into isolated particles and/or smaller clusters, referred to as fragments. When instead shear forces are much weaker compared to the resultant interparticle force responsible for holding

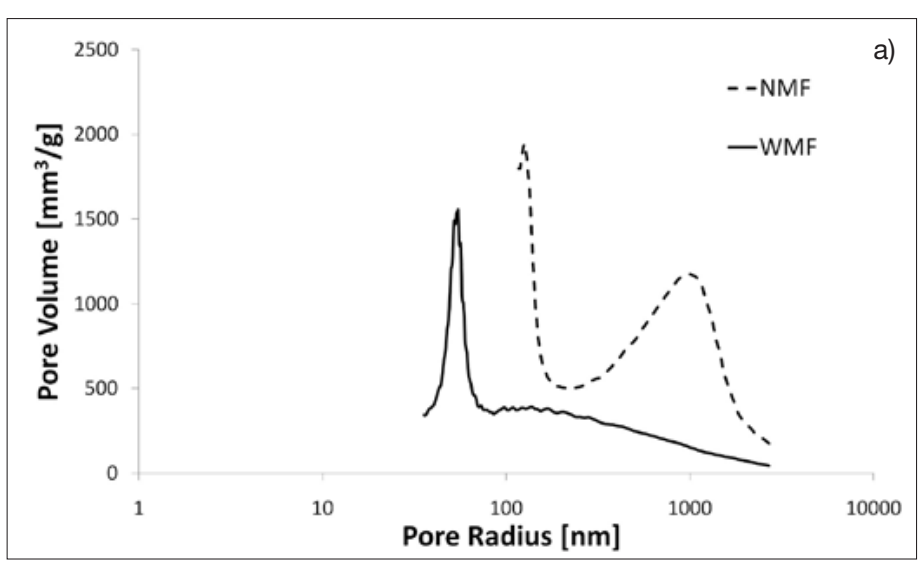

Fig. 3. Pore size distribution from mercury porosimetry (a) and mechanical compression (b) of silica gels obtained from a sol-gel process with magnetic nanocolloids in the absence (NMF) and in the presence (WMF) of an external magnetic field.

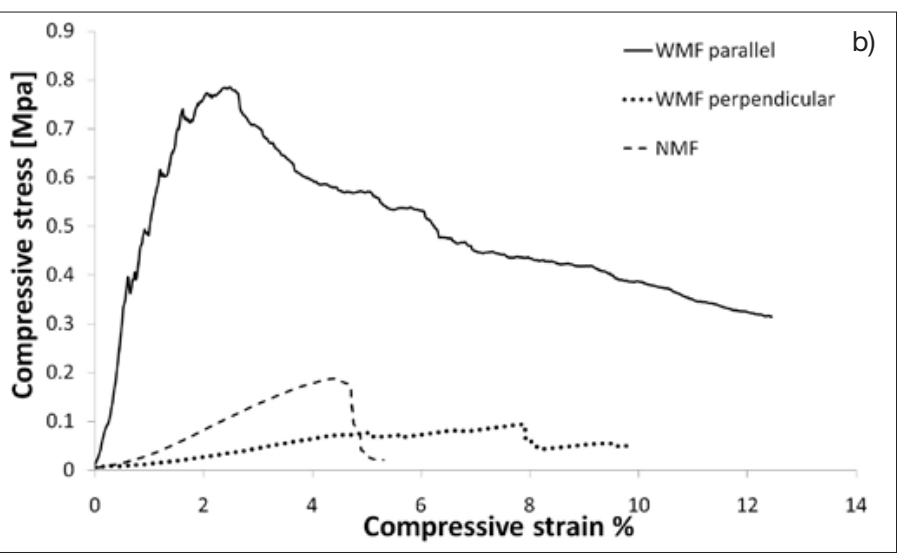


the particles together, a cluster will behave as a rigid body. More interestingly, when shear forces are intermediate but not strong enough to break interparticle bonds, clusters might remain intact but they will be restructured by the action of the flow.

We started by taking a look at how clusters with the same mass but different fractal dimension can be disassembled by the action of the flow. All simulations have been performed for the same values of interparticle interactions, taken as polystyrene spheres. The details can be found in the literature. ${ }^{[12]}$ Fig. 4 shows how the average cluster radius of gyration, normalized by the initial one, as well as how the number of fragments produced evolve as a function of a dimensionless time, obtained by multiplying the physical time by the shear rate. Two types of clusters are considered: very open ones, with fractal dimension $d_{f}=1.8$, (Fig. 4a) and much denser ones having $d_{f}$ $=2.5$ (Fig. 4b). We can immediately recognize a few important features. Open clusters are very rapidly broken into multiple fragments, until a steady state is reached. This steady state value decreases as the rate increases, as expected, and the number of fragments that are formed increases with the shear rate. Moreover the dynamics of this evolution becomes faster at higher shear rates. In the case of denser clusters, the behavior is somewhat similar at high shear rate, even though the number of fragments formed and the reduction of size are smaller than for the open cluster case, but in a similar fashion the dynamics becomes faster at higher shear rate. But what is very different is the behavior of dense clusters at lower shear rates. No significant extent of breakage is observed, as testified by the almost constant number of clusters. However, the time evolution of the cluster size shows an oscillatory behavior. A similar behavior is observed under all conditions in which clusters are exposed to shear rates that are not sufficient to break them,

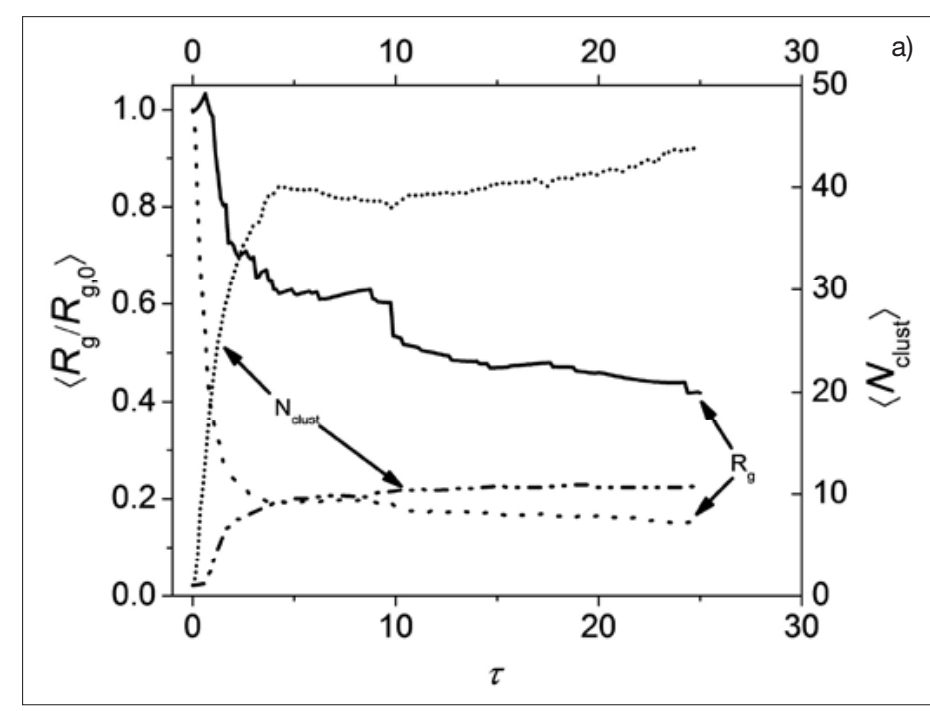

Fig. 4. Dimensionless radius of gyration and number of fragments of a cluster with 100 particles and fractal dimension 1.8 (a) and 2.5 (b) as a function of dimensionless time $\tau$ : dotted lines, 100 $\mathrm{Pa}$; dash-dotted and continuous lines, $25 \mathrm{~Pa}$.

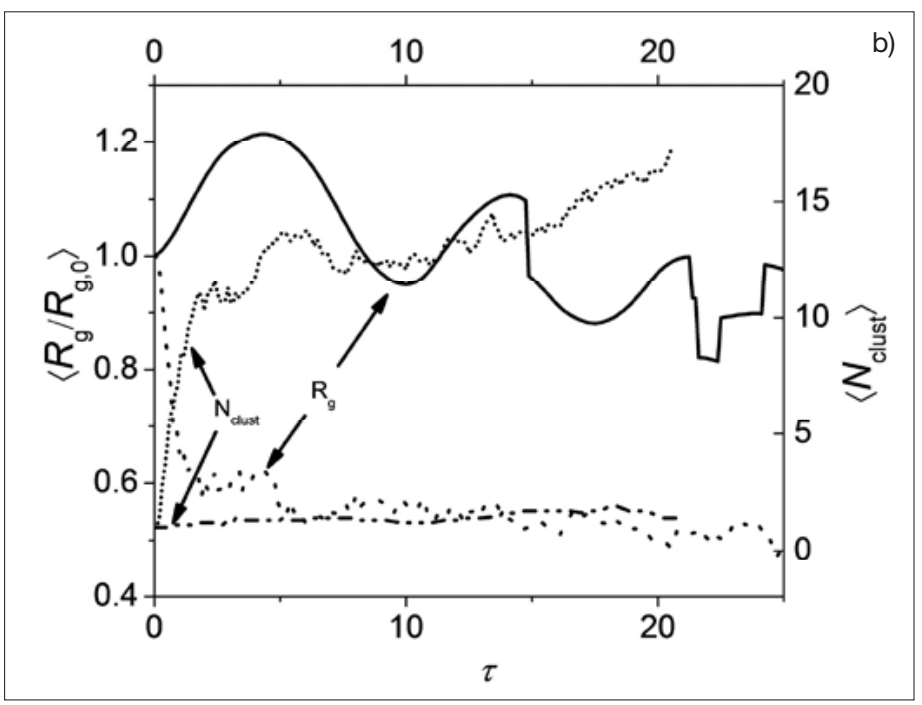

but are not too far from the critical conditions. An even clearer example of that is provided in Fig. 5, showing the time evolution of the size of a smaller cluster with open structure, together with snapshots of its morphology. What happens is that the clusters are restructured by the fluid. When exposed to simple shear a cluster starts ro-

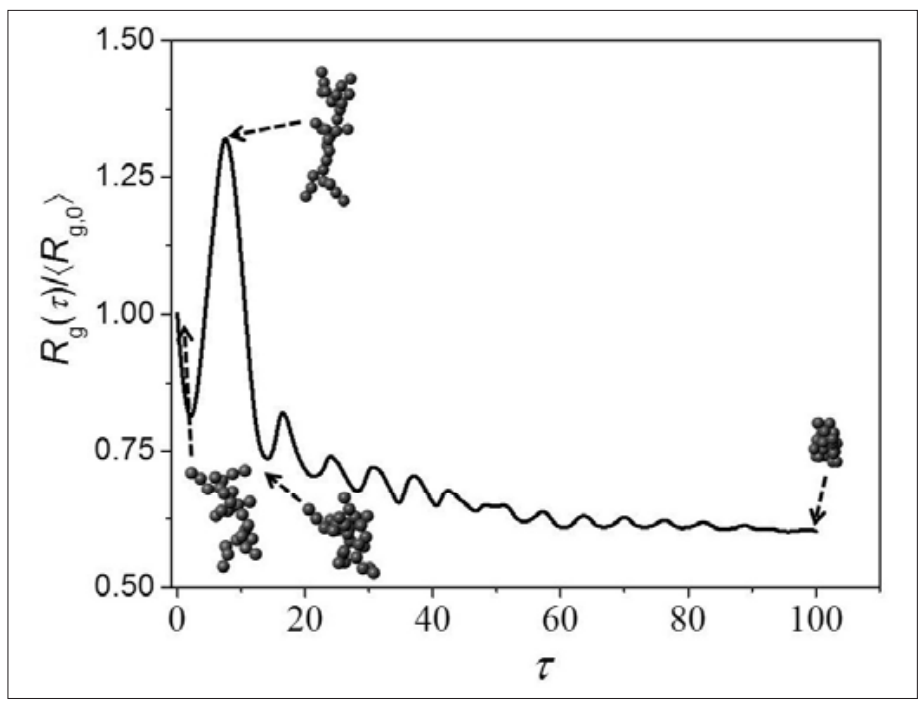

Fig. 5. Time evolution of the dimensionless radius of gyration of a cluster with fractal dimension 1.8 and 30 particles under low shear stress conditions (15 Pa). tating due to the torque imposed by the fluid, and is also initially stretched by the fluid. This stretching phase is followed by a compression phase, because the cluster rotates and the direction in which it was initially stretched has changed orientation and corresponds now to a direction where the fluid is compressing the cluster. The process continues, but as the cluster keeps on rotating and its structure is rearranged, a higher number of bonds per each particle is formed, and the cluster becomes denser and even more resistant to breakup by the shear forces. This phenomenon explains why the average cluster radius oscillates before reaching a steady state.

In general, when a cluster is disassembled or simply restructured, the process reaches a steady state when the fragments formed have the right combination of size and density (i.e. fractal dimension) that allows them to survive in the exposed shear conditions. It is therefore instructive to look at how the average coordination number per particle, i.e. the average number of nearest neighbor particles per average particle in the cluster, evolves in time. Fig. 6 shows exactly this aspect, and demon- 


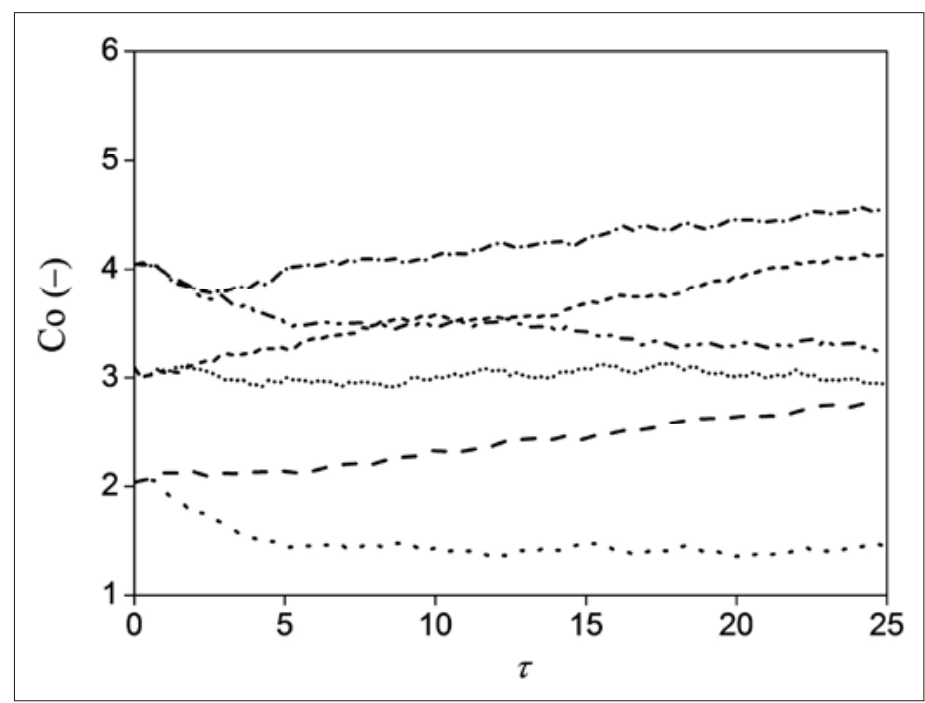

Fig. 6. Coordination number of clusters with 100 particles as a function of dimensionless time $\tau$ : lowest dotted line, $d_{f}$ $=1.8,100 \mathrm{~Pa}$; dashed line, $d_{f}=1.8,25 \mathrm{~Pa}$; small dotted line, $d$ $=2.5,100 \mathrm{~Pa}$; small dashed line, $d_{f}=2.5$ $25 \mathrm{~Pa}$; dash double dotted line, $d_{t}=3$ $100 \mathrm{~Pa}$; dash dotted line, $d_{f}=3,25 \mathrm{~Pa}$

strates that high and low shear rates have a markedly different effect on the overall morphology. At high shear rates, disassembly dominates, leading to a decrease in the coordination values from the initial value, which is expected because small fragments will have in average more particles at the surface, and consequently fewer bonds. On the other hand, at lower shear rate, even though open clusters break more than dense ones, the trend is to increase steadily the number of bonds. This is not too surprising, since it indicates that moderate shear rates are more prone to promote restructuring rather than breakage.

Another important piece of information is given by how the fractal dimension of the cluster evolves as a function of time. This is shown in Fig. 7, from which it can be recognized that there is a threshold value of $d_{f}$ that has a great relevance. In fact, clusters with low fractal dimension tend to become denser until they reach a value of $d_{f}$ close to 2.5 . No matter which conditions are chosen, values higher than 2.5 are not

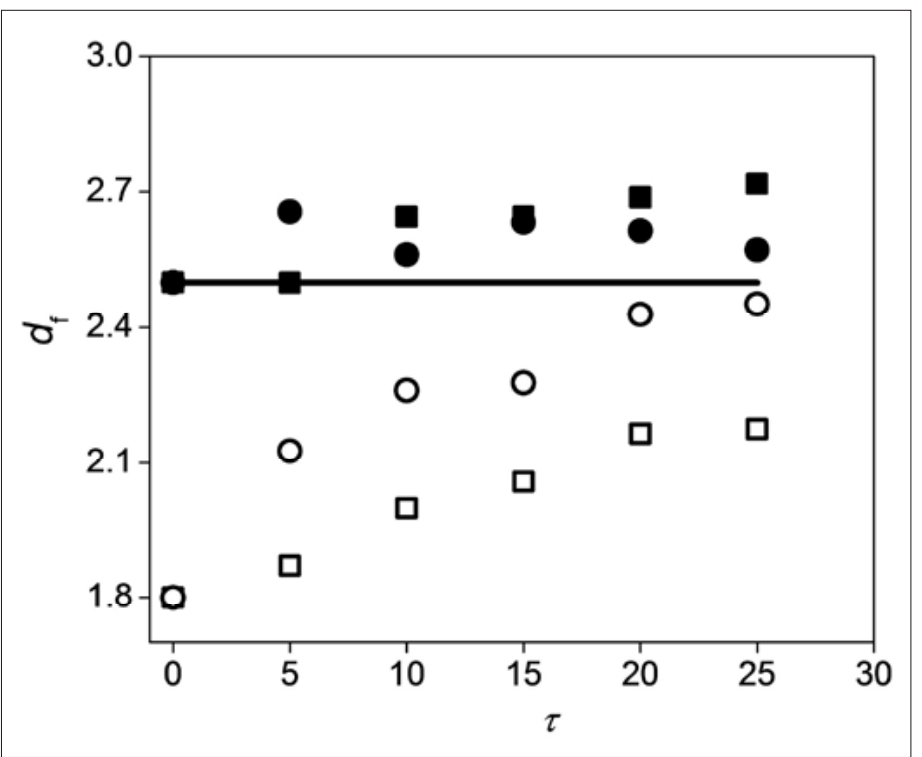

obtained by simple restructuring. On the other hand, clusters that have higher fractal dimension values to begin with tend to preserve it or slightly increase it. The observed behavior is also cluster mass independent. Experiments confirm these findings. ${ }^{[12]}$

A final observation can be made about the fragment mass distribution. Prediction of how clusters are broken is one of the most challenging problems in modeling the flocculation behavior of particles under shear. Our simulations indicate that when clusters with the same morphology but different mass are subject to shear conditions such that the overall force acting on them is the same, similar fragment mass distribution is observed. This is important, because it allows one to make accurate predictions of the behavior of large clusters, which cannot be easily simulated with the technique discussed here. dimension of clusters with 100 particles as a function of time: empty circles, $d_{f}=$ 1.8, 100 Pa; empty squares, $d_{f}=1.8,25$ $\mathrm{Pa}$; filled circles, $d$ squares, $d_{f}=2.5$, $25 \mathrm{~Pa}$.
Fig. 7. Fractal $=2.5,100 \mathrm{~Pa}$; filled

\section{Conclusions}

In this work we have shown two very different examples of how external fields can affect the assembly and disassembly of colloidal nanoparticles. The first case deals with using magnetic fields to manipulate the structure of porous materials obtained in one case from the direct assembly of magnetic nanocolloids, in the second case by using the nanocolloids as directing agents to prepare strongly anisotropic ceramic materials. The properties of the materials, both mechanical and structural (i.e. their porosity) can be very strongly affected by the application of magnetic fields during their preparation, demonstrating how the capability of turning on and off dipolar interaction can lead to dramatic change in the behavior of magnetic colloids. The second example is a computational investigation of how flow fields can interact with clusters of particles. In this case, understanding the effect of the interactions is the primary goal. Through the use of Stokesian Dynamic simulations, we are able to understand under which conditions flow fields disassemble clusters, we can predict how interparticle interactions and cluster morphology are affecting the final distribution of fragments, as well as following the kinetics of fragmentation and restructuring processes.

Controlling and understanding the interactions of nanoparticles with external fields can be greatly advantageous for many applications, but remains a challenging task, often requiring a combination of both experimental and modeling approaches. What is at stake is to learn and acquire the capability to guide the assembly and disassembly of nanoparticles on demand into the form that suits best for a given application.

\section{Acknowledgments}

The authors would like to acknowledge the Swiss National Science Foundation (grants \# 200020-132843 and 200020-126487/1) for financial support, Dr. Frank Krumeich for the help with the SEM pictures, Dr. Kirill Feldman for the help with compression tests and Dr. Josef Kaufmann for the help with mercury porosimetry.

Received: July 5, 2011

[1] G. L. Hornyak, H. F. Tibbals, J. Dutta, J J. Moore, 'Introduction to Nanoscience \& Thechnology', CRC Press, Boca Raton (FL), 2009.

[2] a) J. V. Barth, G. Costantini, K. Kern, Nature 2005, 437, 671; b) J. H. Fendler, Chem. Mater. 1996, 8, 1616; c) K. J. M. Bishop, C. E. Wilmer, S. Soh, B. A. Grzybowski, Small 2009, 5, 1600; d) Z. H. Nie, A. Petukhova, E. Kumacheva, Nature Nanotechnol. 2010, 5, 15.

[3] a) P. J. Lu, E. Zaccarelli, F. Ciulla, A. B. Schofield, F. Sciortino, D. A. Weitz, Nature 2008 453, 499; b) M. Y. Lin, H. M. Lindsay, D. A. 
Weitz, R. C. Ball, R. Klein, P. Meakin, Nature 1989, 339, 360; c) O. D. Velev, A. M. Lenhoff, Curr. Opin. Colloid Interface Sci. 2000, 5, 56.

[4] a) M. Lattuada, T. A. Hatton, Nano Today 2011, 6, 286; b) C. E. Snyder, M. Ong, D. Velegol, Soft Matter 2009, 5, 1263.

[5] R. C. Hayward, D. A. Saville, I. A. Aksay, Nature 2000, 404, 56.

[6] a) M. Furlan, B. Brand, M. Lattuada, Soft Matter 2010, 6, 5636; b) M. Lattuada, M. Furlan, A Butte, M. Morbidelli, Chimia 2009, 63, 78; c) R. E. Rosensweig, 'Ferrohydrodynamics', Dover Publications, 1997.

[7] M. Soos, A. S. Moussa, L. Ehrl, J. Sefcik, H. Wu, M. Morbidelli, J. Colloid Interface Sci. 2008, 319, 577.

[8] R. Massart, Ieee Trans. Magnetics 1981, 17, 1247.

[9] L. J. Gauckler, T. Graule, F. Baader, Mater. Chem. Phys. 1999, 61, 78.

[10] H. Minakuchi, K. Nakanishi, N. Soga, N. Ishizuka, N. Tanaka, Anal. Chem. 1996, 68, 3498 .
[11] a) G. Bossis, J. F. Brady, J. Chem. Phys. 1989, 91, 1866; b) J. F. Brady, G. Bossis, Ann. Rev. Fluid Mech. 1988, 20, 111.

[12] Y. M. Harshe, M. Lattuada, M. Soos, Langmuir 2011, 27, 5739.

[13] J. P. Pantina, E. M. Furst, Phys. Rev. Lett. 2005, 94.

[14] V. Becker, H. Briesen, Phys. Rev. E 2008, 78

[15] P. A. Cundall, O. D. L. Strack, Geotechnique 1979, 29, 47.

[16] Y. M. Harshe, L. Ehrl, M. Lattuada, J. Colloid Interface Sci. 2010, 352, 87.

[17] A. Ahniyaz, Y. Sakamoto, L. Bergstrom, Proc. Nat. Acad. Sci. USA 2007, 104, 17570.

[18] B. A. Grzybowski, C. E. Wilmer, J. Kim, K. P. Browne, K. J. M. Bishop, Soft Matter 2009, 5, 1110 .

[19] L. Ehrl, M. Soos, M. Lattuada, J. Phys. Chem. B 2009, 113, 10587 . 